\title{
Possible Future Developments
}

\author{
Klaus Ueberreiter
}

It is always speculative to throw a glance into the future. In the area of autologous fat, it has been seen that many application possibilities, which are today being "newly" discovered, had already been tried out in the 1920s and the 1930s of the previous century. The really new area of interest is dealing with stem cells from fat tissue. Embryonic stem cells were earlier the focus of intensive research. Then, for example, stem cells were obtained from the bone marrow for the treatment of osteoarthritis. But in recent years, fat tissue has been discovered to contain a virtually inexhaustible source of mesenchymal stem cells. Now the focus has shifted to which type of cells can be developed from this with suitable research projects being conducted and clinical applications being carried out worldwide.

Another broad field is the marking of stem cells with tissue markers, in order to be able to use them finally in an objective manner in the treatment of cancer.

One really cannot predict as to which of these therapies will be successful in hospitals and can be integrated into daily routine. But it is difficult to imagine that the mesenchymal stem cells would not turn out to be useful in hospitals in the long term.

One idea that is presently being considered is that fat tissue can at some time be prophylactically suctioned and the stem cells contained in it can be cryo-conserved. This way, in case of a therapeutic emergency (e.g., acute myocardial infarction), exactly these cells will be available for use. The bankink of embryonic stem cells is being actively propagated in the USA by certain firms, which have specialized in the preservation of tissue in banks, while pointing out possible legal consequence in case of failure of this procedure. Since there are until now no clinical applications for this, promoting this type of conservation appears to be mainly commercially based.

The simple transplantation of fat cells into other body regions has already become daily practice and is being increasingly entrusted to the surgeon, be it for modeling; for the procurement of new sliding tissues, for example, in tendon surgery; for the alleviation of pain in osteoarthritis; in the treatment of chronic fistula and fissures; and in many other indications.

\footnotetext{
K. Ueberreiter $(\bowtie)$

Park-Klinik Birkenwerder, Fachklinik für Plastische und Ästhetische Chirurgie, Birkenwerder, Germany

e-mail: dr@ueberreiter.com
} 